\title{
Absorption characteristics of multi-layered material anechoic coating on frustum-of-a-cone cavities
}

\author{
Liu Guo-qiang ${ }^{1,2}$, Lou Jing-jun ${ }^{1,2}$, Li Hai-tao ${ }^{1,2}$ \\ ${ }^{1}$ College of Power Engineering, Naval Univ. Of Engineering, Wuhan 430033, China \\ 2 National Key Laboratory on Ship Vibration \&Noise; Wuhan 430033, China
}

\begin{abstract}
Improve the low-frequency acoustic performance of sound absorption coating of submarine is a hot issue. Based on the theory of sound wave normally impinging on the absorption layer of 2D,the $2 \mathrm{D}$ shaft mode of frustum-of-a-cone cavities is established by finite element COMSOL, which is test to analyze the absorption characteristics combined structural parameters. The calculation results indicate that under the condition of the same perforation rate ,the absorption performance of multi-layered material anechoic coating is better than that of single-layered material anechoic coating, especially to the performance of low-frequency; the absorption performance of frustum-of-a-cone cavities is better than that of cylindrical and ellipsoidal structures. With the perforation rate is increasing,the absorption performance of anechoic coating in low-frequency improve..Choosing the appropriate cavity structure is important. And the future acoustic optimization of the multi-layered frustum-of-a-cone cavities anechoic coating is guided by the study.
\end{abstract}

\section{Introduction}

Underwater anechoic coating is an important part of the stealth of underwater vehicle. With the development of active sonar technology, the frequency of detecting acoustic is getting lower and lower, so improve the low-frequency acoustic performance of sound absorption coating of submarine is a hot issue, and continuous optimization of the cavity structure could make the anechoic coating in low-frequency perform more excellent [1].

Mainly from the two aspects of the structure and material optimization to increase the absorption performance of anechoic coating in low-frequency. In terms of structure, Shang Chao [2] held that the absorption property of frustum-of-a-cone cavities which make air as backing by using finite element method, according to optimizing the design, the anechoic coating performance of the whole frequency band, especially low-frequency, is further improved; Tao Meng [3] analyzed the absorption property of anechoic coating of the combined cavity type by using waveguide finite element method, the absorption performance of anechoic coating in low-frequency greatly improve with the different embedding ratio of combined cavity type; Tang Weilin [4] put forward that using the boundary conditions of the front and back boundaries the expressions of resonance frequencies and reflection coefficients of coating are given. In terms of materials, Lü Linmei [5] gave the optimization method of how to improve the absorption effect in low-frequency, according to embedded viscoelastic absorption of different shapes of the scattering layer.

The above literature is the optimization of the structure and material of single-layered material anechoic coating. This paper focuses on the analysis of absorption performance of multilayer material of frustum-of-a-cone cavities structure. Results show that the absorption performance of multi-layered material anechoic coating is better than that of single-layered material of frustum-of-a-cone cavities structure and single cavity structure of multi-layer materials. Especially, the absorption effect in low-frequency is improved and obtain the rule about the influence of other structural parameters on the covering layer.

\section{Theoretical model}

Assuming that the fluid is an ideal fluid,it has the characteristics of non viscous, uniform, incompressible flow and zero flow rate. In addition, the density and pressure are assumed to be constant too.

Fluid finite element equation:

$$
\left[M^{p}\right]\{\ddot{p}\}+\left[K^{p}\right]\{p\}+\rho_{0}[R]\{\ddot{\delta}\}=\{\Phi\}
$$

where: $\rho_{0}$ density of fluid medium

$$
\begin{aligned}
& p-\text { acoustic pressure } \\
& {\left[K^{p}\right]-\text { global stiffness matrix; }} \\
& {\left[M^{p}\right]-\text { mass matrix; }} \\
& {[R]-\text { global coupling matrix; }}
\end{aligned}
$$


$\{\Phi\}$ the influence of outer surface acoustic field in an unbounded fluid domin exerted by sound field within a finite domin.

The finite element vibration equation of the general solid structure can be expressed by the following formula [6]:

$$
\left[M^{s}\right]\left\{\ddot{\vec{\delta}}_{V I}\right\}+\left[K^{s}\right]\left\{\left\{\vec{\delta}_{V I}\right\}\right\}=\left\{F^{m}\right\}+\left\{F^{p}\right\}
$$

where $\left[M^{s}\right]$ globa mass matrix;

$\left[K^{s}\right]$ global stiffness matrix 。

$$
\left\{\begin{array}{l}
{\left[M^{s}\right]=\iiint_{V_{I I}^{e}}\left[B_{\delta}\right]^{T}[D]\left[B_{\delta}\right] d V} \\
{\left[K^{s}\right]=\rho_{s} \iiint_{V_{I I}^{e}}\left[N_{\delta}\right]^{T}\left[N_{\delta}\right] d V}
\end{array}\right.
$$

where $\rho_{S}$ density of structural materials;

$\left[N_{\delta}\right]-$ displacement interpolation function matrix;

$[D]$ constitutive relation matrix, $[\sigma]=[D][\varepsilon]$ 。 combine with the boundary conditions,get:

$$
\left[M^{s}\right]\{\ddot{\delta}\}+\left[K^{s}\right]\{\delta\}-\{R\}^{T}\{p\}=\left\{F^{m}\right\}
$$

According to the finite element equation of fluid and solid, the fluid and solid coupling finite element equations of the whole system are rewritten as follows:

$$
\left[\begin{array}{cc}
-[R]^{T} & {\left[K^{s}\right]-\omega^{2}\left[M^{s}\right]} \\
{\left[K^{p}\right]-\left[C_{\Phi}\right]-\omega^{2}\left[M^{p}\right]} & -\rho_{0} \omega^{2}[R]
\end{array}\right]\left\{\begin{array}{l}
p\} \\
\{\delta\}
\end{array}\right\}=\left\{\begin{array}{l}
\left\{F^{m}\right\} \\
\left\{C_{0}\right\}
\end{array}\right\}
$$

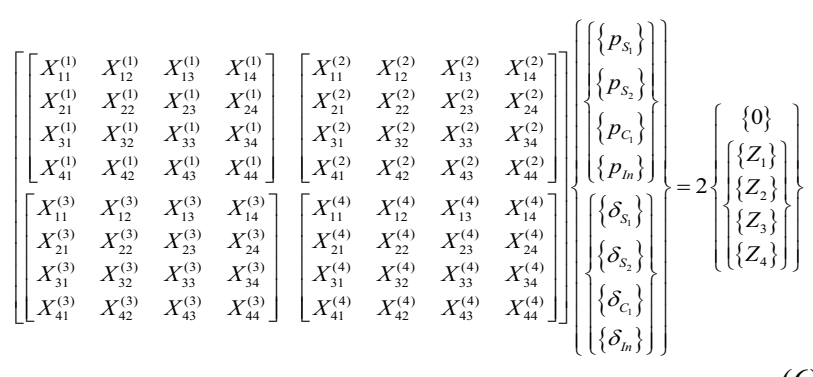

The sound pressure $\mathrm{P}$ of fluid nodes and the displacement of structural nodes are in accordance with the periodic boundary conditions as follows:

$$
\left\{\begin{array}{l}
\left\{\chi_{S_{3}}\right\}=\left\{\chi_{S_{1}}\right\} e^{j \psi_{x}} \\
\left\{\chi_{S_{4}}\right\}=\left\{\chi_{S_{2}}\right\} e^{j\left(\psi_{d x}+\psi_{y}\right)} \\
\left\{\chi_{C_{2}}\right\}=\left\{\chi_{C_{1}}\right\} e^{j \psi_{x}} \\
\left\{\chi_{C_{3}}\right\}=\left\{\chi_{C_{1}}\right\} e^{j\left(\psi_{d x}+\psi_{y}\right)} \\
\left\{\chi_{C_{4}}\right\}=\left\{\chi_{C_{1}}\right\} e^{j\left(\psi_{x}+\psi_{d x}+\psi_{y}\right)}
\end{array}\right.
$$

In the equation of fluid structure, according to the corresponding nodes of the element, each matrix is divided into blocks. And then combined with the boundary conditions, the coupling equations can be changed into the following form:

$\mathrm{X}$ represents delta or $\mathrm{P}$

$\vec{\delta} \_$displacement vector of structure particle

By solving (7), the displacement and sound pressure of each node in the unit can be obtained. The sound pressure is expressed in sum of series respectively.

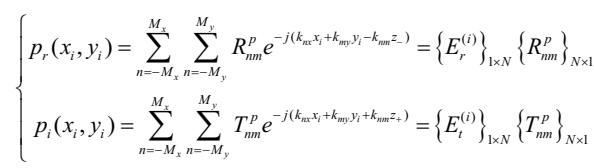

$$
\begin{aligned}
& (i=1,2,3, \cdots \cdots, N)
\end{aligned}
$$

where

$$
\begin{aligned}
& \left\{E_{r}^{(i)}\right\}=\left\{e^{-j\left(k_{n x} x_{i}+k_{m y} y_{i}-k_{n m} z_{-}\right)}\right\} \\
& \left\{E_{t}^{(i)}\right\}=\left\{e^{-j\left(k_{n x} x_{i}+k_{m y} y_{i}+k_{n m} z_{+}\right)}\right\} \\
& \left(n=-M_{x}: 1: M_{x} ; m=-M_{y}: 1: M_{y}\right)
\end{aligned}
$$

From the point of view of the calculation of acoustic energy, The reflection or transmission coefficient can be expressed as follow:

$$
\left\{\begin{array}{l}
R=\sqrt{\sum_{n} \sum_{m} R_{n m}^{2}} / N \\
T=\sqrt{\sum_{n} \sum_{m} T_{n m}^{2}} / N
\end{array}\right.
$$

So expression of sound absorption coefficient is defined as follow:

$$
\alpha=1-|R|^{2}-|T|^{2}
$$

\section{Computational model}

Finite element simulation COMSOL model is shown in Fig. 1. Consider that the arrangement of the absorption performance of frustum-of-a-cone cavities has a periodic, select a unit of anechoic coating as the research object in order to facilitate the calculation. The anechoic coating is composed of a surface layer, a perforated layer, a base layer and a steel backing layer. Fig. 2 is a cross-sectional view of a frustum-of-a-cone cavity structure.

According to the symmetry, the model of the two dimensional axisymmetric model is used in this paper. As shown in Figure 1, on the boundary conditions, using COMSOL to transform the periodic boundary of the 3D model into the boundary of the two dimensional axisymmetric model base on the sound solid coupling module. Due to contact with the vehicle shell, the lower boundary displacement is zero, and the radial displacement of the model is zero because of symmetry, so boundary 6, 7 displacement is zero, the model can only axial deformation; oundary 2, 3, 4 for free deformation 
for simulate the change of cavity with sound pressure. Then, the plane wave is normal incident at the boundary 4, boundary 8,5 set to the specified displacement, boundary 1 is set to a fixed constraint. Triangular mesh that be free splited are adopted when simulation calculation, the maximum size of the grid is $2.0 \mathrm{~mm}$. The sound absorption coefficient of the model is calculated by the sound solid coupling module. Material parameters are shown in Table 1.

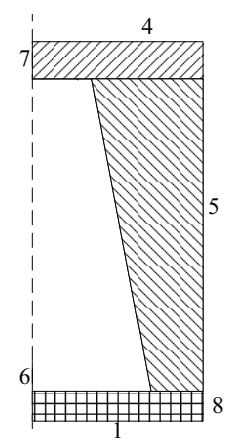

Figure 1. two dimensional axisymmetric model

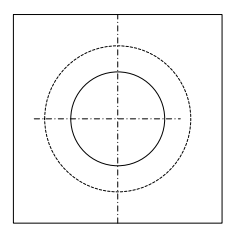

Figure 2. Frustum-of-a-cone cavity structure Table 1 Structure and material parameters of anechoic coating

\begin{tabular}{|c|c|c|c|c|c|c|}
\hline Material & $R / \mathrm{mr}$ & $H / \mathrm{mr}$ & $\rho /(k g \cdot n$ & & $E / \mathrm{P}$ & \\
\hline Surface layer & 21 & 5 & 1100 & $\begin{array}{l}0 . \\
23\end{array}$ & 200 & $\begin{array}{l}0.4 \\
92\end{array}$ \\
\hline $\begin{array}{l}\text { Perforated } \\
\text { layer } \\
\text { (frustum-of } \\
\text {-a-cone } \\
\text { cavity) }\end{array}$ & 12 & 45 & 1300 & $\begin{array}{l}0 . \\
45\end{array}$ & 300 & $\begin{array}{l}0.4 \\
93\end{array}$ \\
\hline Base layer & 21 & 5 & 1400 & $\begin{array}{l}0 . \\
6\end{array}$ & 500 & $\begin{array}{c}0.4 \\
90\end{array}$ \\
\hline Water layer & 21 & 100 & & & & \\
\hline
\end{tabular}

\section{Verification of model validation and analysis of results}

\subsection{Verification of COMSOL model}

In order to verify th e validity of the finite element model, the structure parameters of the viscoelastic model are as follows: outside the cylindrical radius a is $20 \mathrm{~mm}$, the inner radius of $\mathrm{B}$ is $12 \mathrm{~mm}$, and the thickness of $\mathrm{L}$ is $50 \mathrm{~mm}$, the thickness of the surface layer and the base layer are $3 \mathrm{~mm}$. The material parameters of the single layer are as follows: the elastic modulus is $400 \mathrm{MPa}$, the loss factor is 0.2 , the density is $1300 \mathrm{~kg} / \mathrm{m} 3$, and the Poisson's ratio is 0.490. Comparison of analytical formulas for the calculation of the two dimensional theory under the condition of steel backing of Ref.[7] and results of FEM is shown in Fig. 5. In the calculation of the cavity cover cone, frustum-of-a-cone cavity with cylindrical cavity structure is very similar, so in the finite element analysis, the free boundary is added in the cavity, let sound solid coupling boundary and non- reflective fluid sound absorbing boundary are not affected,so does with the the accuracy of the COMSOL model [10]. Through the analysis of the graph, it can be seen that the variation trend and the numerical value of the two curves are consistent within the scope of engineering error, which shows the feasibility of the COMSOL model of the frustum-of-a-cone cavity.

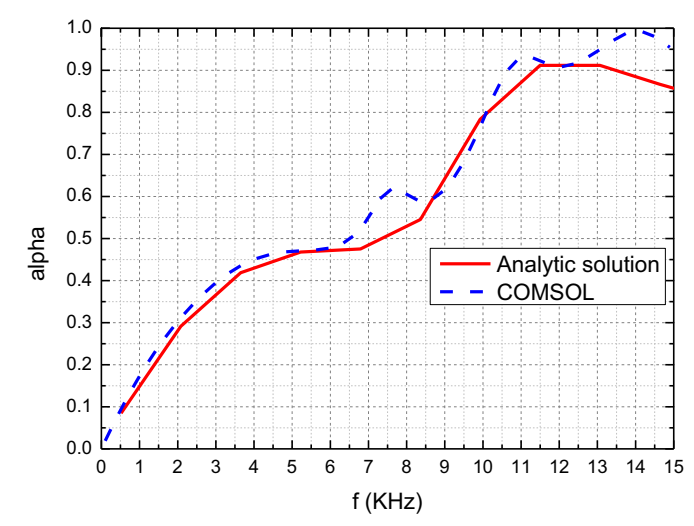

Figure 4. Comparison between two dimensional axisymmetric model and two dimensional theoretical model

\subsection{Effect of materials on anechoic coating}

Fig. 6 shows the effect of different layers of material and the cavity on the sound absorption coefficient or not under the same perforation ratio. The multi-layered materials are composed of these three different materials :the surface layer, the perforated layer, and the base layer ; a single layer of material is a covering of a material. From the graph, the multi-layered materials coatingis better than the single layered material coating. In low-frequency performance, the sound absorption coefficient of multi-layered material is 0.65 , and the sound absorption coefficient of single layered material is 0.59 at the first sound wave crest. With the combination of different materials, the covering layer performs better at low-frequency. With the addition of the cavity, the cover layer is greatly improved at low-frequency sound absorption. That because the perforated layerp is added to the cavity, which make the plane longitudinal wave be converted to shear wave in the process of sound absorption and shear lossfactor of absorption layer is much larger than that of longitudinal wave,w hich can increase disspating the acoustic energy. Besides, the recent published articles [8]-[10] show that multi-layer materials are more efficient than the single-layer ones in terms of stress distribution. Therefore, using multi-layered material instead of single-layer materials could be a good choice for enhancing both absorption and structural performance. 


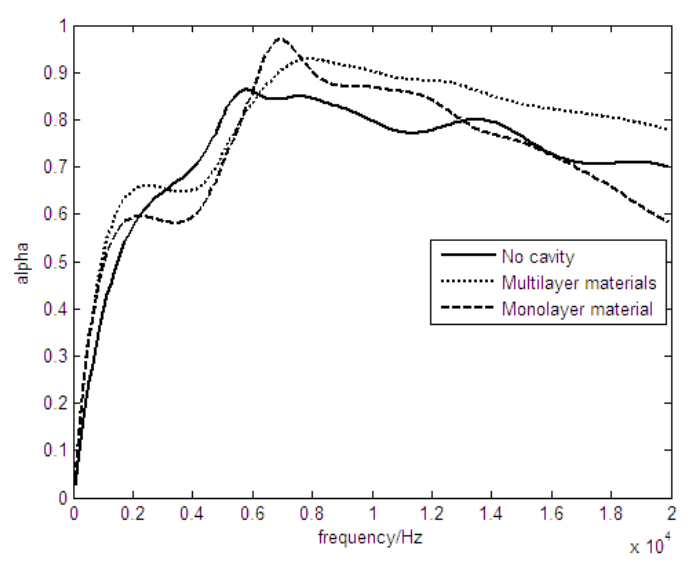

Figure 6. Comparison of sound absorption curves between different layers of material

\subsection{Effect of different cavity structure on anechoic coating}

The influence of cavity structure on sound absorption coefficient under the same perforation rate is discussed in this article. Fig. 7shows that omparison of sound absorption curves between cylindrical cavity, frustum-of-a-cone cavity, hybrid cavity and ellipsoidal cavity. Obviously, the low- frequency sound absorption performance of the hybrid cavity is better than that of the other cavity structures,and frustum-of-a-cone cavity is second. frustum-of-a-cone cavit in the first absorption trough much lower than the cylindrical cavity, ellipsoid cavity, which sound absorption coefficient is 0.49 and corresponding frequency is $3950 \mathrm{~Hz}$. In the middle and high frequency, sound absorption performance of the four kinds of cavity structures are generally the same, and sound absorption coefficient is stable at about 0.8. In engineering application, two kinds of situations should be taken into account to choose.

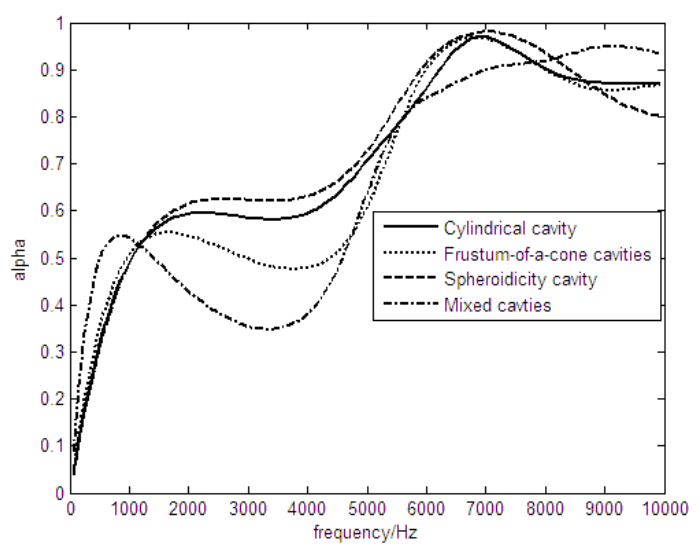

Figure 7. Comparison of sound absorption curves between different cavity structures

\subsection{Influence of perforation rate on anechoic coating}

This example discusses the influence of the change of the perforation rate on anechoic coating. In Fig. 8, The perforation rates of case 1,case 2,case 3 are
$28 \%, 46 \%, 60 \%$.As known from Fig. 8, With the perforation rate is increasing, the absorption performance of anechoic coating in low-frequency improve, the first sound wave crest to the low frequency shift. In the middle and high frequency, the sound absorption effect of the covering layer of the three kinds of perforation rate structure is generally similar. With perforation rate increases ,The lifting effect for the medium- frequency and high- frequency sound absorption is very small.

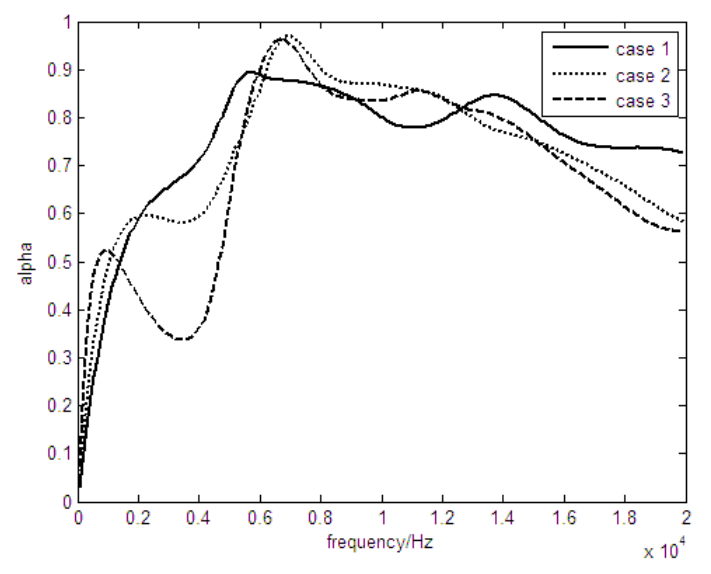

Figure 8. Comparison of sound absorption curves between different perforation rates.

\section{Conclusion}

In view of relatively poor situation of the absorption performance of anechoic coating in low-frequency currently, This paper, based on the theory of sound wave normally impinging on the absorption layer of $2 \mathrm{D}$, build the $2 \mathrm{D}$ shaft mode of frustum-of-a-cone cavities which is established by finite element COMSOL, and analyze the absorption characteristics combined structural parameters, conclusions are as follows:

(1) Under the condition of the same perforation rate, the absorption performance of multi-layered material anechoic coating is better than that of single-layered material anechoic coating, especially to the performance of low-frequency; the absorption performance of frustum-of-a-cone cavities play an important role in improving the absorption performance in low-frequency of anechoic coating, which is better than that of single cavity structure.

(2) The perforation rate is essential for improving the absorption performance in low-frequency of anechoic coating. With the perforation rate is increasing,the absorption performance of anechoic coating in low-frequency improve. The relationship between the absorption performance in interm ediate-frequency/ high-frequency and the perforation rate is not very clos

(3) Choosing the appropriate cavity structure is important. When compared to combined cavity structure, frustum-of-a-cone cavitie structure provide little assistance to the improvement of the absorption performance of anechoic coating in low-frequency; compared with the cylindrical and ellipsoidal form, the opposite is the case. 


\section{References}

1. MENG Xiao-yu,XIAO Guo-lin,CHEN Hong.Review of the present situation and development of acoustic stealth technology for submarines abroad.J.Ship Science and Technology 33,11(2011).

2. SHANG Chao,ZHANG Jia-zhong,WEI Ying-jie.Absorption characterstics of anechoic coating embedding frustum-of-a-cone cavities.J.Journal of Ship Mechanics 14,12 (2010).

3. TAO Meng,ZHUO Lin-kai.Acoustic performance of sound absorption coating containing composite cavities.J.Journal of Shanghai Jiaotong University 47,3 (2013).

4. TANG Wei-lin,HE Shi-ping,FAN Jun.Two-dimensional model for acoustic absorption of viscoelastic coating containing cylindrical holes .J.Acta Acustica 30,4 (2005).

5. Lü Lin-Mei,Wen Ji-Hong,Zhao Hong-Gang,et al.Dynamical mechanical property of viscoelastic materials and its effect on acoustic absorption of
coating.J.Acta Phys.Sin 63,15(2014).

6. HU Peng.Research of Acoustic Coating's Properities under Hydrostatic Pressure by Modular-FEM Method[D].Shanghai:Shanghai Jiaotong University, 2008.

7. HE Shi-ping,TANG Wei-lin,HE Lin,et al.Analysis of acoustic characteristics of anechoic coating containing varying sectional cylindrical cavity .J.Journal of Ship Mechanics 10,1 (2006).

8. M.M. Mirsayar, B. Takabi.Fracture of underwater notched structures.J. Engineering Solid Mechanics 4 (2016) .

9. B. Takabi.Thermomechanical transient analysis of a thick-hollow FGM cylinder.J. Engineering Solid Mechanics 4 (2016) .

10. [10] SHANG Chao,WEI Ying-jie,ZHANG Jia-zhong,CAO Wei.Absorption characteristics of anechoic coating embedding elliptic-cyclinder cavities.J.Journal of Harbin Institute of Technology 44,1 (2012). 